\title{
Eficacia de la infiltración de ozono paravertebral lumbar y en puntos gatillos como coadyuvante del tratamiento en pacientes con dolor lumbar crónico y lumbociatalgia crónica en el síndrome doloroso miofascial aislado o acompañado de otras patologías
}

\author{
E. Silva Jiménez, M. Toro y C. Baíz \\ Servicio de Medicina Física y Rehabilitación. Hospital Universitario De Los Andes. Clínica del Dolor. \\ Instituto Médico La Floresta. Venezuela
}

Silva Jiménez E, Toro M, Baíz C. Eficacia de la infiltración de ozono paravertebral lumbar y en puntos gatillos como coadyuvante del tratamiento en pacientes con dolor lumbar crónico y lumbociatalgia crónica en el síndrome doloroso miofascial aislado o acompañado de otras patologías. Rev Soc Esp Dolor 2014; 21(1): 23-38.

\begin{abstract}
Background: After the first episode of low back pain, recurrence persists for a year or more in 25 to $60 \%$, affecting economically active population, causing disability and $80 \%$ absenteeism.

Objective: To evaluate the effectiveness of the use of ozone injection technique with paravertebral lumbar and trigger points along the drug treatment and rehabilitation in patients with chronic low back pain and sciatica due to chronic pain syndrome myofascial isolated or accompanied other pathologies.

Methods: A non-probability, experimental type double-blind controlled. We studied 43 patients (22 experimental and 21 control). The experimental group received lumbar paravertebral ozone trigger points and more drug treatment and rehabilitation. The control group received drug treatment and rehabilitation. Was applied in both groups VAS, Oswestry and measuring the degrees of flexion of the trunk at the beginning, two and four weeks after initiation of therapy.

Results: The application of ozone and lumbar paravertebral and trigger points along the drug treatment and rehabilitation in the management of patients with chronic low back pain and sciatica, compared to just drug treatment and rehabilitation, was more effective, with statistical significance $(\mathrm{p}<$ $0.05)$ to decrease the intensity of pain $(90.5 \%)$, functional
\end{abstract}

Recibido: 11-10-12.

Aceptado: 15-01-13. disability (90.5\%) and increased degrees of trunk flexion (85.7 $\%$ versus 40.0, 70 and $75 \%$, respectively, at four weeks after initiation of treatment. It is evident improvement in patients with MDS or accompanied isolated lateral recess syndrome, facet syndrome, disc herniation varying degrees except the central extrusion.

Conclusions: The ozone infiltration paravertebral lumbar and trigger points along the drug treatment and rehabilitation was more effective, to reduce pain and functional disability and increase the degrees of trunk flexion early in patients with chronic low back pain and sciatica due to chronic isolated or accompanied by other conditions that cause back pain.

Key words: Low back pain and chronic sciatica. Myofascial pain syndrome (MPS). Ozone therapy. Paravertebral. Trigger points.

\section{RESUMEN}

Introducción: Posterior al primer episodio de dolor lumbar, la recurrencia persiste durante un año o más en el 25 al $60 \%$, afectando a población económicamente activa, causando discapacidad y en $80 \%$ ausentismo laboral.

Objetivo: Evaluar el grado de eficacia del uso de la técnica de infiltración con ozono paravertebral lumbar y en puntos gatillos junto al tratamiento farmacológico y rehabilitador, en pacientes con dolor lumbar crónico y lumbociatalgia crónica debido al síndrome doloroso miofascial (SDM) aislado o acompañado de otras patologías.

Métodos: Estudio no probabilístico, de tipo experimental controlado, doble ciego. Se estudiaron 43 pacientes (22 experimental y 21 control). El grupo experimental recibió ozono paravertebral lumbar y en puntos gatillos más tratamiento farmacológico y rehabilitador. El grupo control recibió tratamiento farmacológico y rehabilitador. Se aplicó en ambos grupos la escala de EVA, Oswestry y la medición de los grados de flexión del tronco al inicio, dos y cuatro semanas posterior al comienzo del tratamiento. 
Resultados: La aplicación de ozono paravertebral lumbar y en puntos gatillos, junto al tratamiento farmacológico y rehabilitador, en el manejo de pacientes con dolor lumbar y lumbociatalgia crónica, comparado con solo tratamiento farmacológico y rehabilitador, resultó ser más eficaz, con significancia estadística $(p<0,05)$ para disminuir la intensidad del dolor $(90,5 \%)$, la incapacidad funcional $(90,5 \%)$, y aumentó los grados de flexión del tronco (85,7 \% versus 40,0, 70 y $75 \%$ ) respectivamente, a las cuatro semanas posteriores al inicio del tratamiento. Se evidencia mejoría en pacientes con SDM aislado o acompañado de síndrome de receso lateral, síndrome facetario, grados variables de hernia discal excepto la extrusión central.

Conclusiones: La infiltración de ozono paravertebral lumbar $y$ en puntos gatillos junto al tratamiento farmacológico y rehabilitador resultó ser más eficaz, para disminuir el dolor e incapacidad funcional y aumentar los grados de flexión del tronco precozmente en pacientes con dolor lumbar crónico y lumbociatalgia crónica debido al SDM aislado o acompañado de otras patologías que originan dolor lumbar.

Palabras clave: Dolor lumbar crónico y lumbociatalgia crónica. Síndrome doloroso miofascial (SDM). Ozonoterapia. Paravertebral. Puntos gatillos.

\section{INTRODUCCIÓN}

El dolor lumbar constituye el segundo motivo de baja laboral tras el resfriado común y el motivo de consulta más frecuente del aparato locomotor, tanto en la atención primaria como en la especializada (1). La aparición frecuente del dolor lumbar, aunque en su mayoría es autolimitado, puede tener tendencia a la cronicidad, convirtiéndolo en un problema de salud personal con grandes repercusiones laborales, económicas, sociales, familiares y de salud mental (2), situación que empobrece la calidad de vida del paciente y lo aleja cada vez más del cumplimiento de sus actividades previas. Algunos estudios refieren que el dolor lumbar crónico es la causa del 75-80 \% del ausentismo laboral, razón por la cual se plantean alternativas de solución a este padecimiento. La Organización Mundial de la Salud (3) incluyó al dolor lumbar como una prioridad en la década de los huesos y articulaciones, en vista de la cantidad de personas afectadas. Por otra parte los costos médicos, laborales y de incapacidad por dolor lumbar a nivel mundial se estiman en 50.000 millones de dólares anuales, por lo que no solamente representa un problema de salud pública para la multitud de individuos, sino a la sociedad en su conjunto.

Es conocido que alrededor de un $10 \%$ del dolor lumbar se convierte en crónico y predispone a la discapacidad. Sin embargo actualmente se cree que las cifras son mayores, ya que después de un primer episodio de dolor lumbar se presenta recurrencia durante un año o más en el 25 a $60 \%$ de los pacientes (4).
Una de las principales causas de dolor lumbar son las lumbalgias inespecíficas presentes en el $85 \%$ de los pacientes, acompañada de contractura de los músculos paravertebrales o paraespinales (5). El síndrome doloroso miofascial (SDM) se caracteriza por una banda tensa palpable del músculo o de su fascia que representa un espasmo segmentario de la porción del músculo, también por la presencia de un punto gatillo activo o latente que al presionarla origina un patrón de dolor específico para cada músculo, que no tiene la distribución clásica de una raíz nerviosa y no exhibe déficits sensitivos o motores asociados (6). La hipótesis más aceptada del SDM es una sobrecarga muscular inicial, debido a gran uso o traumatismo que causa una disfunción de la placa motora que se traduce en una excesiva liberación de acetilcolina, una despolarización prolongada con contracción sostenida del músculo, lo que lleva a la compresión de los vasos sanguíneos pequeños, isquemia tisular local, liberación de bradicinina y excitación de nociceptores (7). Si la tensión inicial, generadora del acortamiento, el dolor y el espasmo no se trata, el cuadro se cronifica y como consecuencia de ello, el músculo se acorta más, se hace menos elástico, más limitado, desencadenando un dolor difícil de tratar.

El SDM puede presentarse como única causa de dolor lumbar crónico o acompañar a otras etiologías como el síndrome facetario o compresión radicular (por diferentes grados de discopatías, síndrome de receso lateral, estenosis del canal vertebral).

Es posible que estos puntos gatillos miofasciales no se diagnostiquen por desconocimiento de muchos especialistas $y$, en ocasiones, estos pacientes evolucionan peor que aquellos con diagnóstico de otras causas de dolor lumbar (8).

El ozono $\left(\mathrm{O}_{3}\right)$ utilizado en medicina es una mezcla de $95 \%$ oxígeno con $5 \%$ de ozono $\left(\mathrm{O}_{2} \mathrm{O}_{3}\right)(9)$ que libera un átomo de oxígeno al ponerse en contacto con los tejidos generando, a nivel intramuscular, un efecto antiinflamatorio debido al aumento de la producción de enzimas antioxidantes que lleva a un estado de adaptación al estrés oxidativo, neutralizando la formación de compuestos inestables de oxígeno reactivo que son productos tóxicos liberados por la salida del núcleo pulposo, responsables en parte de la inflamación del nervio (10). El efecto analgésico del ozono hace disminuir la contractura muscular de defensa que, de forma refleja, se activa para proteger la zona de la hernia discal. Según Borrelli (11) esta acción está relacionada con la inhibición de la síntesis de prostaglandinas y citocinas proinflamatorias y aumenta la liberación de citocinas inmunosupresoras. La oxigenación localizada y la analgesia permiten la vasodilatación y la relajación del músculo, favoreciendo la oxidación del lactato, neutralizando la acidosis, incrementando la síntesis de ATP, la homeostasis del calcio y la reabsorción del edema (9). 
El objetivo de la investigación consiste en evaluar el grado de eficacia del uso de la técnica de infiltración con ozono intramuscular paravertebral lumbar y en puntos gatillos, junto al tratamiento farmacológico y rehabilitador, en pacientes con dolor lumbar crónico y lumbociatalgia crónica debido al SDM aislado o acompañado de otras patologías que originan dolor lumbar. Se comprueba así de forma objetiva, del gran abanico de patologías asociadas a este padecimiento, en cuáles la técnica en estudio ofrece beneficios reales.

\section{MATERIALES Y MÉTODOS}

Se realizó una investigación no probabilística de tipo experimental, comparada con un grupo control, doble ciego.

Del universo de pacientes con dolor lumbar crónico y lumbociatalgia crónica referidos al Servicio de Medicina Física y Rehabilitación del Hospital Universitario De Los Andes (Venezuela), se distribuyeron en orden secuencial posterior a su evaluación, para pertenecer a cada uno de los grupos. Se estudiaron 43 sujetos en total (22 grupo experimental y 21 grupo control) en el periodo comprendido entre junio a diciembre de 2011.

\section{Criterios de inclusión}

- Pacientes masculinos y femeninos.

- Edades comprendidas entre los 18 y 65 años.

- Pacientes con clínica de dolor lumbar crónico o lumbociatalgia crónica de tres o más meses de evolución y con intensidad del dolor (EVA igual o superior a 4).

- Se incluyeron los pacientes con diagnósticos de síndrome doloroso miofascial, síndrome facetario, compresión radicular por diferentes grados de discopatías o por síndrome de receso lateral.

- Pacientes con patología lumbar confirmada por imagen de resonancia magnética de columna lumbosacra.

\section{Criterios de exclusión}

- Cirugía de columna lumbar previa.

- Presencia imagenológica de otras patologías en la región lumbosacra (espondilolistesis, espondilolisis, fractura de columna lumbar, lesión ocupante de espacio, escoliosis lumbar $>20^{\circ}$, estenosis del canal vertebral).

- Deseos por parte del paciente de obtener informe de incapacidad laboral.

\section{Procedimiento}

Bajo consentimiento informado, el grupo experimental o estudio conformado por 22 pacientes recibió infiltración con ozono intramuscular paravertebral lumbar y en puntos gatillos, junto al tratamiento farmacológico y rehabilitador, durante 4 semanas. Mientras el grupo control conformado por 21 pacientes recibió sólo la combinación de tratamiento farmacológico y rehabilitador.

\section{Técnica de infiltración de ozono paravertebral lumbar y en puntos gatillos}

Se aplicaron 2 infiltraciones semanales durante cuatro semanas ( 8 infiltraciones en total), siguiendo el protocolo del Dr. Tabaracci (12).

1. Se coloca al paciente en decúbito prono, con un cojín a nivel abdominal.

2. Se palpa la cresta iliaca, sugestivo del nivel de la apófisis espinosa L4; luego se dirige al espacio interespinoso correspondiente a la patología herniaria.

3. Se practica la infiltración paravertebral lumbar, a $2 \mathrm{~cm}$ tomados lateralmente de la apófisis espinosa lumbar, con una inclinación de $45^{\circ}$, de forma bilateral.

4. Se introduce la aguja muy lentamente hasta encontrar la resistencia de la fascia muscular.

5. Se aplicará aspiración repetida durante la técnica, para evitar inyectar en vasos sanguíneos y linfáticos.

6. Se introducen $10 \mathrm{ml}$ de ozono $\left(\mathrm{O}_{3}\right)$ por punto, a una concentración de $20 \mathrm{mcg} / \mathrm{ml}$.

7. Después de la práctica de la infiltración, se realiza un masaje suave con la mano abierta para favorecer la difusión del gas y se deja descansar al paciente en posición supina durante 30 minutos, para controlar los efectos vagales (sudoración, bradicardia, hipotensión).

8. Se aplica crioterapia en la región lumbar durante 15 minutos, posterior a la infiltración con oxígeno-ozono.

Los puntos gatillos dolorosos ubicados en otras áreas de la zona lumbar como en la musculatura de miembros inferiores relacionada con el recorrido del nervio ciático, también fueron infiltrados con un volumen entre 5-10 ml dependiendo del sitio anatómico y la concentración entre 10 y $20 \mathrm{mcg} / \mathrm{ml}$.

\section{Tratamiento farmacológico}

El grupo experimental y el grupo control se manejaron con tratamiento farmacológico, sin variación de este entre ellos.

En caso de pacientes con dolor lumbar crónico se indicó:

- Acemetacina $\left(\right.$ Pranex $^{\circledR}$ ) en cápsulas de 90 mg, OD durante 7 días. 
- Paracetamol (Agurin ${ }^{\circledR}$ ) en comprimidos de $1 \mathrm{~g}$ cada 12 horas, durante 7 días.

- Tizanidina $\left(\right.$ Sirdalud $^{\circledR}$ ) en comprimidos de 2 mg OD, durante 7 días.

Los pacientes de ambos grupos que presentaron lumbociatalgia crónica se les adicionó al tratamiento anterior el uso de Pregabalina (Lyrica ${ }^{\circledR}$ ) en cápsulas de 75 mg BID por un mes.

El tratamiento lo adquirieron los pacientes de forma gratuita, sin ningún coste, con el fin de garantizar su cumplimiento.

\section{Tratamiento rehabilitador}

Se realizó de la siguiente manera:

- Escuela de espalda: constituida por una clase teórica en donde se explicó al paciente, de una forma simple, la anatomía de la columna vertebral, las causas frecuentes del dolor lumbar y las medidas ergonómicas necesarias para evitar futuras recaídas. Se realizó una segunda clase de tipo práctica, para enseñarle al paciente la forma correcta de realizar los ejercicios de estiramientos y fortalecimientos de abdominales y del raquis. Por último se le entregó una guía explicativa con dibujos del contenido de ambas clases.

- Terapia fisica: constituida por el uso de medios físicos y la realización de ejercicios cinco días a la semana, durante cuatro semanas. Entre los agentes físicos se utilizaron cada uno durante 20 minutos: el hotpacks, la estimulación eléctrica nerviosa transcutánea (TENS), la diatermia a 4 watts- $\mathrm{cm}^{2}$ y masajes en la zona lumbar. Los ejercicios realizados fueron los de Williams, McKenzie y los ejercicios de estabilización espinal (isométricos de co-contracción del transverso del abdomen y multífidos).

\section{Parámetros}

- Se utilizó la escala de EVA (mide la intensidad del dolor, referida por el paciente).

- Escala de Oswestry (mide el porcentaje de incapacidad funcional \% IF del paciente); utiliza 10 ítems cada uno con 6 alternativas. Se categorizan los resultados en 5 renglones de \% IF: mínima (0-19 puntos); moderada (20-39 puntos); intensa (40-59 puntos); discapacidad (60-79 puntos); y máxima (80-100 puntos).

- Medición de los grados de flexión del tronco utilizando el goniómetro. Los resultados se categorizan en diferentes grados. Grado I: flexión del tronco entre $0-30^{\circ}$; grado II: flexión del tronco entre $31-50^{\circ}$; grado III: flexión del tronco entre $51-70^{\circ}$; y grado IV: flexión del tronco entre $71-90^{\circ}$. A mayores grados de flexión del tronco que presente el paciente representa mejor movilidad de la región lumbar.
La medición de estos tres parámetros se realizó al inicio del tratamiento y a las 2, 4, 6 semanas posteriores al comienzo del mismo y fue llevado a cabo por personal médico distinto al que administraba el tratamiento, por lo que no sabían el grupo al cual pertenecía el paciente.

\section{Análisis estadístico}

Para el análisis estadístico descriptivo de las variables cualitativas se utilizaron las cifras absolutas y relativas representadas en tablas de distribución de frecuencia, mientras que para las variables cuantitativas se utilizaron medidas de tendencia central, de dispersión y de distribución. Así mismo, para conocer si el último tipo de variables en referencia se distribuían en forma normal, se revisaron las curvas de distribución de probabilidades, de quintiles y la prueba de Kolmogorov-Smirnov para la muestra.

Para la relación entre variables cuantitativas se emplearon pruebas de comparación de medias. En este caso, como se realizaron varias mediciones en el tiempo a un mismo grupo de sujetos, se utilizó la prueba $t$ de Student para muestras pareadas cuando las variables de interés se distribuyeron de forma normal. En el caso de las variables cuantitativas que no se distribuyeron de forma normal, la comparación de los grupos se realizó con la prueba estadística no paramétrica de Wilcoxon.

Para la comparación de las variables cualitativas, es decir, proporciones de mejorías por categorías, igualmente se empleó la prueba de Wilcoxon. Para todas las actividades descritas en este apartado del análisis estadístico, se contó con el apoyo de un programa informático para el análisis de datos, denominado como SPSS ${ }^{\circledR}$, versión 17.0.

\section{RESULTADOS}

De la población estudiada con dolor lumbar y lumbociatalgia crónica, el sexo femenino fue el predominante en un $60,5 \%(\mathrm{n}=26)$. La edad promedio fue de 45,8 \pm DE 8,1 años (rango 26-62 años); de los cuales el 44,3\% $(n=19)$ de los pacientes tenía entre 40-49 años, seguido por el 30,2 \% $(n=13)$ con edades entre 50-59 años, y un 20,9\% $(n=9)$ entre 30-39 años. De acuerdo a los diferentes tipos de ocupación se pudo observar que en primer lugar se ubica al personal de enfermería con un 18,5\% $(\mathrm{n}=8)$, seguido por las personas que se dedican a los oficios del hogar, con un $16,3 \%(n=7)$ y el personal secretarial con el 14,0\% $(n=6)$. El dolor lumbar crónico fue la forma de presentación clínica más frecuente de la enfermedad en la población estudiada, presentándose en el $65,1 \%$ de los pacientes $(n=28)$, seguido de la lumbociatalgia crónica con un $34,9 \%(n=15)$. 
Según la etiología de la enfermedad, el $100 \%$ de los pacientes presentó el componente miofascial en un $39,5 \%(\mathrm{n}=17)$ de forma aislada, mientras que en el $25,6 \%(\mathrm{n}=11)$ se acompañó de síndrome facetario y un $16,3 \%(n=7)$ con protrusión discal (Tabla I).

TABLA I. PATOLOGÍAS ENCONTRADAS EN LOS PACIENTES CON DOLOR LUMBAR CRÓNICO Y LUMBOCIATALGIA CRÓNICA EN LA INVESTIGACIÓN

\begin{tabular}{lcc}
\hline \multicolumn{1}{c}{ Etiología de la enfermedad } & Frecuencia & Porcentaje \\
\hline $\begin{array}{l}\text { Componente miofascial } \\
\text { aislado }\end{array}$ & 17 & 39,5 \\
$\begin{array}{l}\text { Componente miofascial y } \\
\text { síndrome facetario }\end{array}$ & 11 & 25,6 \\
$\begin{array}{l}\text { Componente miofascial y } \\
\text { protrusión discal }\end{array}$ & 7 & 16,3 \\
$\begin{array}{l}\text { Componente miofascial y } \\
\text { síndrome de receso lateral }\end{array}$ & 5 & 11,6 \\
$\begin{array}{l}\text { Componente miofascial y } \\
\text { extrusión discal central }\end{array}$ & 3 & 7,0 \\
Total & 43 & 100,0 \\
\hline
\end{tabular}

Fuente: Formato de Recolección de Datos.

En la tabla II se evidencia que la localización anatómica de los puntos gatillos tiene mayor frecuencia en el área paravertebral en todos los pacientes, es decir, en un $100 \%$. No obstante en los pacientes con dolor lumbar crónico también existen otros puntos dolorosos simultáneamente, localizados a nivel del músculo cuadrado lumbar en $44,18 \%$ y piriforme en $18,60 \%$. En los pacientes con lumbociatalgia crónica también se presentan puntos gatillos en los miembros inferiores, ocupando el primer lugar los gemelos en 18,60\%, seguido de los isquiotibiales en $11,62 \%$ y aductores de cadera en un $7 \%$ de los pacientes.
TABLA II. LOCALIZACIÓN ANATÓMICA DE LOS PUNTOS GATILLOS RELACIONADOS CON EL SDM ENCONTRADOS EN LA INVESTIGACIÓN EN LOS PACIENTES CON DOLOR LUMBAR CRÓNICO Y LUMBOCIATALGIA CRÓNICA

\begin{tabular}{lcc}
\hline \multicolumn{1}{c}{$\begin{array}{c}\text { Localización de puntos } \\
\text { gatillos }\end{array}$} & Frecuencia & Porcentaje \\
\hline Paravertebrales & 43 & 100,0 \\
Cuadrado lumbar & 19 & 44,18 \\
Piriforme & 8 & 18,60 \\
Gemelos & 8 & 18,60 \\
Isquiotibiales & 5 & 11,62 \\
Aductores de cadera & 3 & 7,00 \\
\hline
\end{tabular}

Fuente: Formato de Recolección de Datos.

Los puntos gatillos localizados en el área paravertebral correspondieron a los niveles L4-L5 en $55 \%$, L5-S1 en $30 \%$ y otras áreas en $15 \%$ (Tabla III y Fig. 1).

Se realizaron comparaciones de los niveles basales de los puntajes de los parámetros evaluados con sus respectivas medidas al inicio, a las 2, 4 y 6 semanas de estar recibiendo los correspondientes esquemas de tratamiento. Los pacientes que recibieron el tratamiento de estudio presentaron disminuciones promedio más altas con respecto al puntaje EVA y el porcentaje de IF e incrementos promedios mayores en el GFT. Estos datos se demuestran con alta significancia estadística $(\mathrm{p}<0,05)$.

La mayoría de los pacientes del grupo estudio solo requirieron 4 semanas de tratamiento multimodal para presentar resolución de las manifestaciones clínicas de la patología, en contraste con los pacientes del grupo control que requirieron 2 semanas adicionales, llegando a las 6 semanas o más de tratamiento para presentar los niveles de mejoría alcanzados por los pacientes del grupo de estudio (Tabla IV).

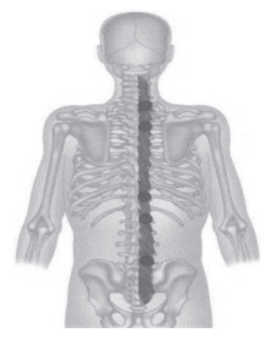

Paravertebrales

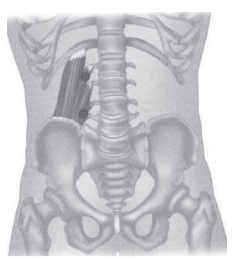

Cuadrado lumbar

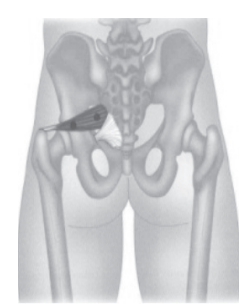

Piriforme

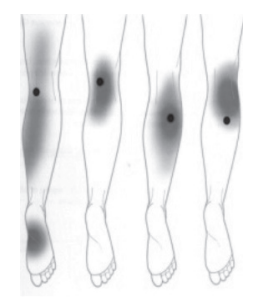

Gemelos

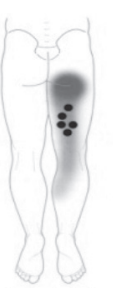

Isquiotobiales

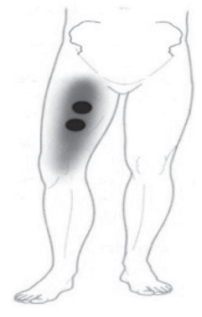

Aductores

Fig. 1. Puntos gatillos encontrados en la investigación en pacientes con dolor lumbar crónico y lumbociatalgia crónica. Ilustraciones tomadas del Libro conciso de los puntos gatillos de Simeon Niel-Asher, 2008. 
TABLA III. EFICACIA DE LA INTERVENCIÓN DE LOS ESQUEMAS DE TRATAMIENTO UTILIZADOS EN EL GRUPO EXPERIMENTAL Y EN EL GRUPO CONTROL, AL EVALUAR LA INTENSIDAD DEL DOLOR (EVA), GRADOS DE FLEXIÓN DEL TRONCO (GFT) Y EL \% DE INCAPACIDAD FUNCIONAL (\% IF)

\begin{tabular}{|c|c|c|c|c|c|c|}
\hline \multicolumn{3}{|c|}{$\begin{array}{c}\text { Grupo experimental } \\
\text { (Ozonoterapia más tratamiento farmacológico y } \\
\text { rehabilitador) }\end{array}$} & \multicolumn{4}{|c|}{$\begin{array}{c}\text { Grupo control } \\
\text { (Tratamiento farmacológico y rehabilitador) }\end{array}$} \\
\hline $\begin{array}{l}\text { EVA - inicio } \\
7,50 \pm 1,14\end{array}$ & $\begin{array}{l}\text { EVA - } 2 \text { semanas } \\
3,27 \pm 1,67 \\
p=0,000000000147^{*}\end{array}$ & $\begin{array}{l}\text { EVA - } 4 \text { semanas } \\
\mathrm{Me}=1 \\
\mathrm{p}=0,0000712^{* *}\end{array}$ & $\begin{array}{l}\text { EVA inicio } \\
6,95 \pm 1,63\end{array}$ & $\begin{array}{l}\text { EVA }-2 \text { semanas } \\
4,67 \pm 1,56 \\
p=0,00000000163^{*}\end{array}$ & $\begin{array}{l}\text { EVA }-4 \text { semanas } \\
3,85 \pm 1,46 \\
p=0,00000000270^{*}\end{array}$ & $\begin{array}{l}\text { EVA - } 6 \text { semanas } \\
2,60 \pm 2,28 \\
\mathrm{p}=0,0000000040^{*}\end{array}$ \\
\hline $\begin{array}{l}\text { GFT- inicio } \\
54,65 \pm 12,62\end{array}$ & $\begin{array}{l}\text { GFT - } 2 \text { semanas } \\
71,14 \pm 8,99 \\
\mathrm{p}=0,00000000145^{*}\end{array}$ & $\begin{array}{l}\text { GFT- } 4 \text { semanas } \\
79,52 \pm 9,86 \\
p=0,00000000274^{*}\end{array}$ & $\begin{array}{l}\text { GFT- inicio } \\
62,43 \pm 10,99\end{array}$ & $\begin{array}{l}\text { GFT- } 2 \text { semanas } \\
74,00 \pm 9,57 \\
p=0,0000000205^{*}\end{array}$ & $\begin{array}{l}\text { GFT - } 4 \text { semanas } \\
75,75 \pm 10,92 \\
\mathrm{p}=0,000000115^{*}\end{array}$ & $\begin{array}{l}\text { GFT - } 6 \text { semanas } \\
76,00 \pm 10,83 \\
\mathrm{p}=0,000000182 *\end{array}$ \\
\hline $\begin{array}{l}\% \text { de IF - inicio } \\
48,36 \pm 14,36\end{array}$ & $\begin{array}{l}\% \text { IF }-2 \text { semanas } \\
\mathrm{Me}=11 \\
\mathrm{p}=0,0000454 * *\end{array}$ & $\begin{array}{l}\% \text { IF }-4 \text { semanas } \\
M e=2 \\
p=0,0000792 * *\end{array}$ & $\begin{array}{l}\% \text { IF - inicio } \\
36,95 \pm 17,05\end{array}$ & $\begin{array}{l}\% \text { IF- } 2 \text { semanas } \\
24,62 \pm 14,87 \\
p=0,00000000419 *\end{array}$ & $\begin{array}{l}\% \text { IF- } 4 \text { semanas } \\
16,65 \pm 15,38 \\
p=0,000000524^{*}\end{array}$ & $\begin{array}{l}\% \text { IF- } 6 \text { semanas } \\
M e=10 \\
p=0,000128^{*}\end{array}$ \\
\hline
\end{tabular}

Fuente: Formato de Recolección de Datos; *Prueba t de Student para muestras pareadas; **Prueba de Wilcoxon; Me: mediana.

TABLA IV. DIFERENCIA DE MEDIAS PARA COMPARAR LA MEJORÍA CLÍNICA CON RESPECTO AL INICIO Y DESPUÉS DE LA APLICACIÓN DE LOS DOS ESQUEMAS DE TRATAMIENTO

\begin{tabular}{|c|c|}
\hline Grupo experimental (diferencia de medias) & Grupo control (diferencia de medias) \\
\hline EVA al inicio versus EVA 2 semanas & EVA al inicio versus EVA 2 semanas \\
\hline $4,23(\mathrm{p}=0,000000000147)$ & $2,29(\mathrm{p}=0,00000000163)$ \\
\hline EVA al inicio versus EVA 4 semanas & EVA al inicio versus EVA 4 semanas \\
\hline $6,29(\mathrm{p}=0,0000712)^{* *}$ & $3,15(\mathrm{p}=0,00000000270)$ \\
\hline GFT al inicio versus GFT 2 semanas & GFT al inicio versus GFT 2 semanas \\
\hline $16,59(\mathrm{p}=0,00000000145)$ & $11,57(\mathrm{p}=0,0000000205)$ \\
\hline GFT al inicio versus GFT 4 semanas & GFT al inicio versus GFT 4 semanas \\
\hline $25,95(\mathrm{p}=0,00000000274)$ & $12,70(p=0,000000115)$ \\
\hline$\%$ IF al inicio versus $\%$ IF 2 semanas & $\%$ IF al inicio versus $\%$ IF 2 semanas \\
\hline $32,68(\mathrm{p}=0,0000454)^{* *}$ & $12,33(\mathrm{p}=0,00000000419)$ \\
\hline$\%$ IF al inicio versus $\%$ IF 4 semanas & $\%$ IF al inicio versus $\%$ IF 4 semanas \\
\hline $40,14(\mathrm{p}=0,0000792)^{* *}$ & $18,85(\mathrm{p}=0,000000524)$ \\
\hline
\end{tabular}

Fuente: Formato de Recolección de Datos; *Prueba t de Student para muestras pareadas; **Prueba de Wilcoxon; EVA: puntos en la escala visual análoga; GFT: grados de flexión del tronco; \% IF: porcentaje de incapacidad funcional.

En todos los casos, al comparar las medidas de desenlace antes y durante el tratamiento, tanto a las 2 como a las 4 semanas, la diferencia de medias fue significativamente mayor en el grupo de pacientes que recibieron el tratamiento experimental con infiltración intramuscular de $\mathrm{O}_{2} \mathrm{O}_{3}$ paravertebral lumbar y en puntos gatillos más tratamiento farmacológico y rehabilitador, comparado con el resultado clínico en los pacientes que recibieron el tratamiento farmacológico y rehabilitador. Es decir, se presentó una mejoría altamente significativa en la percepción al dolor (EVA), en el grado de flexión del tronco (medido con goniómetro) y en el porcentaje de incapacidad funcional (escala de Oswestry) (Tabla V).

Se evidencia que al inicio del tratamiento en los pacientes que recibieron la infiltración intramuscular con ozono más tratamiento farmacológico y rehabilitador, presenta- 
TABLA V. EFICACIA DEL ESQUEMA DE TRATAMIENTO EN EL GRUPO EXPERIMENTAL AL INICIO Y A LAS 2 SEMANAS POSTERIORES AL TRATAMIENTO. COMPARACIÓN DE LAS CATEGORÍAS DEL PUNTAJE DE LA EVA ANTES Y DESPUÉS DE LA INTERVENCIÓN TERAPÉUTICA

Categorías del puntaje de la EVA 2 semanas después de la aplicación del tratamiento

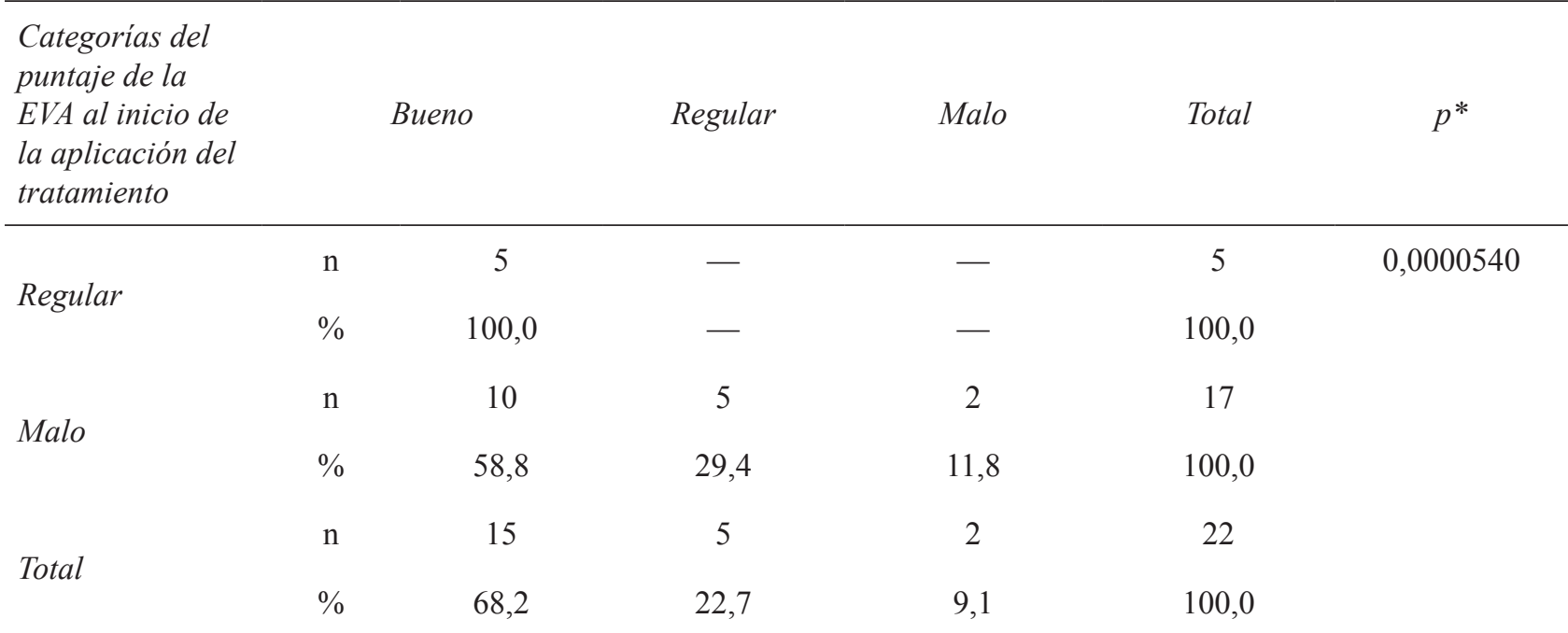

Fuente: Formato de Recolección de Datos; EVA: escala visual análoga; Bueno: grado de dolor entre 0,0 y 3,3 cm; Regular: grado de dolor entre 3,4 y 6,7 cm; Malo: grado de dolor entre 6,8 y 10,0 cm; *Prueba de Wilcoxon.

ron un EVA regular 5 pacientes y malo 17 pacientes, de los cuales a las 2 semanas después de haber iniciado el tratamiento el $100 \%$ de los primeros y el $58,8 \%$ de los segundos evolucionaron a la categoría de bueno, lo cual fue estadísticamente significativo $(\mathrm{p}=0,0000540)$.

En la tabla VI se muestra que al inicio del tratamiento los pacientes que recibieron los tratamientos farmacológico $\mathrm{y}$ rehabilitador presentaron un EVA regular 9 pacientes y malo 12 pacientes, de los cuales a las 2 semanas después de haber iniciado el tratamiento el 55,6\% de los primeros y ninguno $(0 \%)$ de los segundos evolucionaron a la categoría de bueno.

$\mathrm{Al}$ analizar los resultados de las tablas V y VI se puede evidenciar que el tratamiento del grupo experimental fue más eficaz (68,2\% de los pacientes) que el tratamiento del grupo control (con un $23,8 \%$ de los pacientes) en dismi-

TABLA VI. EFICACIA DEL ESQUEMA DE TRATAMIENTO EN EL GRUPO CONTROL AL INICIO Y A LAS 2 SEMANAS POSTERIORES AL TRATAMIENTO. COMPARACIÓN DE LAS CATEGORÍAS DEL PUNTAJE DE LA EVA ANTES Y DESPUÉS DE LA INTERVENCIÓN TERAPÉUTICA

Categorías del puntaje de la EVA 2 semanas después de la aplicación del tratamiento

\begin{tabular}{|c|c|c|c|c|c|c|}
\hline $\begin{array}{l}\text { Categorías del } \\
\text { puntaje de la EVA al } \\
\text { inicio de la aplicación } \\
\text { del tratamiento }\end{array}$ & & Bueno & Regular & Malo & Total & $p^{*}$ \\
\hline \multirow{2}{*}{ Regular } & $\mathrm{n}$ & 5 & 4 & - & 9 & \multirow[t]{6}{*}{$0,000183 *$} \\
\hline & $\%$ & 55,6 & 44,4 & - & 100,0 & \\
\hline \multirow{2}{*}{ Malo } & $\mathrm{n}$ & - & 9 & 3 & 12 & \\
\hline & $\%$ & - & 75,0 & 25,0 & 100,0 & \\
\hline \multirow{2}{*}{ Total } & $\mathrm{n}$ & 5 & 13 & 3 & 21 & \\
\hline & $\%$ & 23,8 & 61,9 & 14,3 & 100,0 & \\
\hline
\end{tabular}


nuir la intensidad del dolor según las categorías de EVA de regular o malo al inicio del tratamiento y evolucionar a la categoría de bueno a las 2 semanas después de haber iniciado el tratamiento.

Analizando la tabla VII evidenciamos que al inicio del tratamiento, los pacientes que recibieron la infiltración intramuscular con oxígeno ozono más tratamiento farmacológico y rehabilitador, presentaron un EVA regular 4 pacientes y malo 17 pacientes, de los cuales a las 4 semanas después de haber iniciado el tratamiento el $100 \%$ de los primeros y el $88,2 \%$ de los segundos evolucionaron a la categoría de bueno.

La tabla VIII muestra que, al inicio del tratamiento, los pacientes que recibieron los tratamientos farmacológico y rehabilitador presentaron un EVA regular 9 pacientes y malo 11 pacientes, de los cuales, a las 4 semanas después de haber iniciado el tratamiento, el $66,7 \%$ de los primeros y sólo el $18,1 \%$ de los segundos evolucionaron a la categoría de bueno.

Analizando los resultados de las tablas VII y VIII vemos que el tratamiento del grupo experimental fue más eficaz en un $90,5 \%$ de los pacientes que el tratamiento del grupo control en un $40,0 \%$ de los pacientes; también fue efectivo en cuanto a la disminución de la intensidad del dolor según las categorías de EVA, de regular o malo al inicio del tratamiento, y evolucionar a la categoría de bueno a las 4 semanas después de haber iniciado el tratamiento, lo que se demuestra con alta significancia estadística $(\mathrm{p}=$ $0,0000579)$.
En la tabla IX se evidencia que al inicio del tratamiento en los pacientes que recibieron la infiltración intramuscular con oxígeno ozono paravertebral lumbar y en puntos miofasciales más tratamiento farmacológico y rehabilitador, presentaron un grado de flexión del tronco grado II, 10 pacientes y grado III, 11 pacientes, de los cuales, a las 2 semanas después de haber iniciado el tratamiento, el $10 \%$ de los primeros y el 90,9\% de los segundos evolucionaron al grado IV de máxima flexión del tronco.

En la tabla $\mathrm{X}$ se puede ver que al inicio del tratamiento los pacientes que recibieron tratamiento farmacológico y rehabilitador presentaron un grado II de flexión del tronco 5 pacientes y un grado III, 11 pacientes, de los cuales a las 2 semanas después de haber iniciado el tratamiento ninguno $(0 \%)$ de los primeros y el $63,6 \%$ de los segundos evolucionaron al grado IV de máxima flexión del tronco.

En la tabla XI se muestra que al inicio del tratamiento los pacientes que recibieron la infiltración intramuscular con oxígeno ozono a nivel paravertebral lumbar y en puntos gatillos más tratamiento farmacológico y rehabilitador, presentaron un grado de flexión del tronco grado II, 10 pacientes, y grado III, 11 pacientes, de los cuales a las 4 semanas después de haber iniciado el tratamiento el $80 \%$ de los primeros y el 90,9\% de los segundos evolucionaron al grado IV de máxima flexión del tronco.

Examinando la tabla XII se evidencia que al inicio del tratamiento los pacientes que recibieron tratamiento farmacológico y rehabilitador presentaron un grado II de flexión del tronco 5 pacientes y grado III, 11 pacientes de

TABLA VII. EFICACIA DEL ESQUEMA DE TRATAMIENTO EN EL GRUPO EXPERIMENTAL AL INICIO Y A LAS 4 SEMANAS POSTERIORES AL TRATAMIENTO. COMPARACIÓN DE LAS CATEGORÍAS DEL PUNTAJE DE LA EVA ANTES Y DESPUÉS DE LA INTERVENCIÓN TERAPÉUTICA

Categorías del puntaje de la EVA 4 semanas después de la aplicación del tratamiento

\begin{tabular}{|c|c|c|c|c|c|}
\hline $\begin{array}{l}\text { Categorías del } \\
\text { puntaje de la } \\
\text { EVA al inicio de } \\
\text { la aplicación del } \\
\text { tratamiento }\end{array}$ & & & Malo & Total & $p^{*}$ \\
\hline \multirow{2}{*}{ Regular } & $\mathrm{n}$ & 4 & - & 4 & \multirow[t]{6}{*}{$0,0000579 *$} \\
\hline & $\%$ & 100 & - & 100 & \\
\hline \multirow{2}{*}{ Malo } & $\mathrm{n}$ & 15 & 2 & 17 & \\
\hline & $\%$ & 88,2 & 11,3 & 100,0 & \\
\hline \multirow{2}{*}{ Total } & $\mathrm{n}$ & 19 & 2 & 21 & \\
\hline & $\%$ & 90,5 & 9,5 & 100,0 & \\
\hline
\end{tabular}

Fuente: Formato de Recolección de Datos; EVA: escala visual análoga; Bueno: grado de dolor entre 0,0 y 3,3 cm; Regular: grado de dolor entre 3,4 y 6,7 cm; Malo: grado de dolor entre 6,8 y 10,0 cm; *Prueba de Wilcoxon.

Nota: un paciente del grupo experimental abandono el tratamiento por hipotensión. 
TABLA VIII. EFICACIA DEL ESQUEMA DE TRATAMIENTO EN EL GRUPO CONTROL AL INICIO Y A LAS 4 SEMANAS POSTERIORES AL TRATAMIENTO. COMPARACIÓN DE LAS CATEGORÍAS DEL PUNTAJE DE LA EVA ANTES Y DESPUÉS DE LA INTERVENCIÓN TERAPÉUTICA

Categorías del puntaje de la EVA 4 semanas después de la aplicación del tratamiento

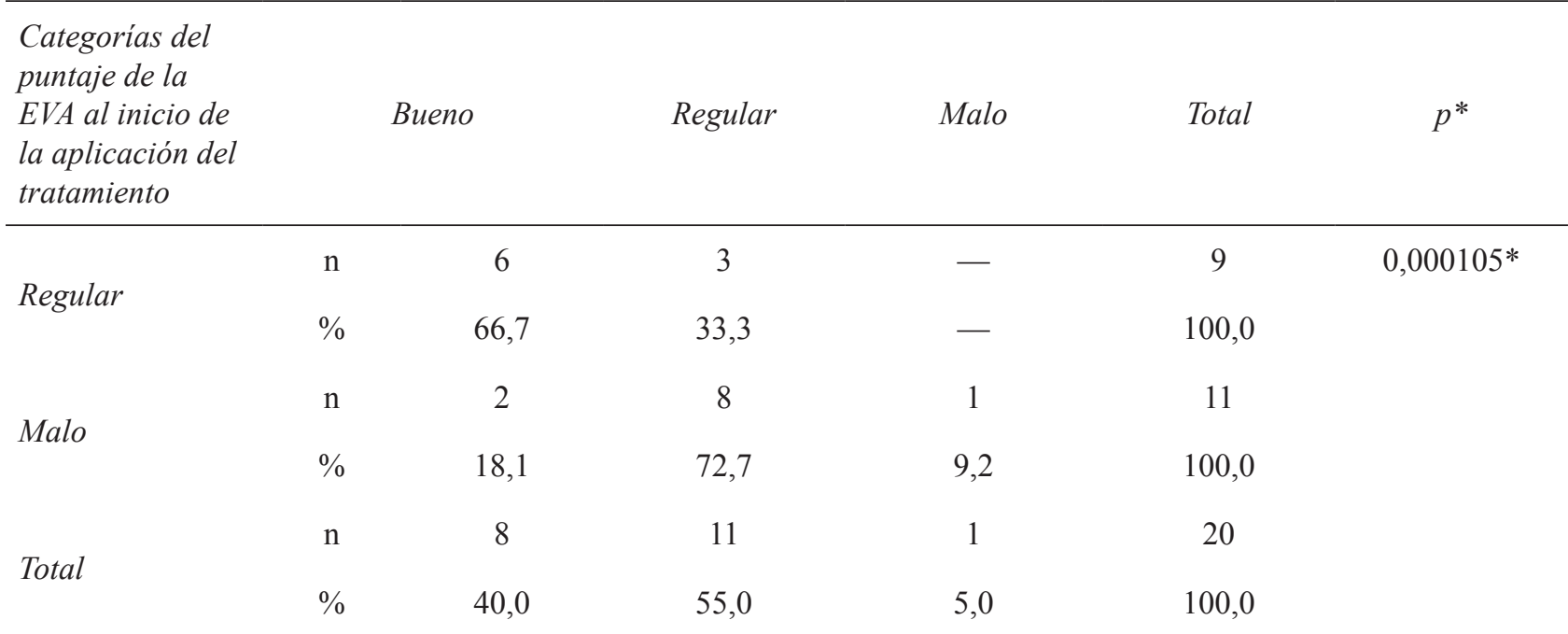

Fuente: Formato de Recolección de Datos; EVA: escala visual análoga; Bueno: grado de dolor entre 0,0 y 3,3 cm; Regular: grado de dolor entre 3,4 y 6,7 cm; Malo: grado de dolor entre 6,8 y 10,0 cm; *Prueba de Wilcoxon.

Nota: un paciente del grupo experimental abandono el tratamiento por domicilio lejano.

TABLA IX. EFICACIA DEL ESQUEMA DE TRATAMIENTO EN EL GRUPO EXPERIMENTAL AL INICIO Y A LAS 2 SEMANAS POSTERIORES AL TRATAMIENTO. COMPARACIÓN DE LAS CATEGORÍAS DE GRADOS DE FLEXIÓN DEL TRONCO ANTES Y DESPUÉS DE LA INTERVENCIÓN TERAPÉUTICA

Categorías del GFT 2 semanas después de la aplicación del tratamiento

\begin{tabular}{lcccccc}
\hline $\begin{array}{l}\text { Categorias del GFT al } \\
\text { inicio de la aplicación } \\
\text { del tratamiento }\end{array}$ & Grado II & Grado III & Grado IV & Total & $p^{*}$ \\
\hline Grado II & $\mathrm{n}$ & 1 & 8 & 1 & 10 & 0,0000201 \\
& $\%$ & 10,0 & 80,0 & 10,0 & 100,0 & \\
Grado III & $\mathrm{n}$ & - & 1 & 10 & 11 & \\
& $\%$ & - & 9,1 & 90,9 & 100,0 & \\
Grado IV & $\mathrm{n}$ & - & - & 1 & 1 & \\
& $\%$ & - & - & 100,0 & 100,0 & \\
Total & $\mathrm{n}$ & 1 & 9 & 12 & 22 & \\
& $\%$ & 4,5 & 40,9 & 54,6 & 100,0 & \\
\hline
\end{tabular}

Fuente: Formato de Recolección de Datos; Grado I: flexión del tronco entre 0-30; Grado II: flexión del tronco entre 31-50; Grado III: flexión del tronco entre 51-70; Grado IV: flexión del tronco entre 71-90; *Prueba de Wilcoxon.

los cuales, a las 4 semanas después de haber iniciado el tratamiento, el $80 \%$ de los primeros y el $81,8 \%$ de los segundos evolucionaron al grado IV de máxima flexión del tronco.
En los resultados de las tablas XI y XII se muestra que el tratamiento del grupo experimental fue más eficaz en un $85,7 \%$ de los pacientes que el tratamiento del grupo control, en un 75,0 \% de los pacientes, en aumentar el grado de 
TABLA X. EFICACIA DEL ESQUEMA DE TRATAMIENTO EN EL GRUPO CONTROL AL INICIO Y A LAS 2 SEMANAS POSTERIORES AL TRATAMIENTO. COMPARACIÓN DE LAS CATEGORÍAS DE GRADOS DE FLEXIÓN DEL TRONCO (GFT) AL INICIO Y DESPUÉS DE LA INTERVENCIÓN TERAPÉUTICA

Categorías del GFT 2 semanas después de la aplicación del tratamiento

\begin{tabular}{lcccccc}
\hline $\begin{array}{l}\text { Categorias del GFT al } \\
\text { inicio de la aplicación } \\
\text { del tratamiento }\end{array}$ & Grado II & Grado III & Grado IV & Total & $p^{*}$ \\
\hline Grado II & $\mathrm{n}$ & 1 & 4 & - & 5 & $0,000911^{*}$ \\
& $\%$ & 20,0 & 80,0 & - & 100,0 & \\
Grado III & $\mathrm{n}$ & - & 4 & 7 & 11 & \\
& $\%$ & - & 36,4 & 63,6 & 100,0 & \\
Grado IV & $\mathrm{n}$ & - & - & 5 & 5 & \\
& $\%$ & - & - & 100,0 & 100,0 & \\
Total & $\mathrm{n}$ & 1 & 8 & 12 & 21 & \\
& $\%$ & 4,8 & 38,1 & 57,1 & 100,0 & \\
\hline
\end{tabular}

Fuente: Formato de Recolección de Datos; Grado I: flexión del tronco entre 0-30; Grado II: flexión del tronco entre 31-50; Grado III: flexión del tronco entre 51-70; Grado IV: flexión del tronco entre 71-90; *Prueba de Wilcoxon.

TABLA XI. EFICACIA DEL ESQUEMA DE TRATAMIENTO EN EL GRUPO EXPERIMENTAL AL INICIO Y A LAS 4 SEMANAS POSTERIORES AL TRATAMIENTO. COMPARACIÓN DE LAS CATEGORÍAS DE GRADOS DE FLEXIÓN DEL TRONCO ANTES Y DESPUÉS DE LA INTERVENCIÓN TERAPÉUTICA

Categorías del GFT 4 semanas después de la aplicación del tratamiento

Categorías del GFT al

inicio de la aplicación

del tratamiento

Grado II Grado III Grado IV Total $\quad p^{*}$

\begin{tabular}{|c|c|c|c|c|c|c|}
\hline \multirow{2}{*}{ Grado II } & $\mathrm{n}$ & 1 & 1 & 8 & 10 & $0,0000793^{*}$ \\
\hline & $\%$ & 10,0 & 10,0 & 80,0 & 100,0 & \\
\hline \multirow{2}{*}{ Grado III } & $\mathrm{n}$ & - & 1 & 10 & 11 & \\
\hline & $\%$ & - & 9,1 & 90,9 & 100,0 & \\
\hline \multirow{2}{*}{ Total } & $\mathrm{n}$ & 1 & 2 & 18 & 21 & \\
\hline & $\%$ & 4,8 & 9,5 & 85,7 & 100,0 & \\
\hline
\end{tabular}

Fuente: Formato de Recolección de Datos; Grado I: flexión del tronco entre 0-30; Grado II: flexión del tronco entre 31-50;

Grado III: flexión del tronco entre 51-70; Grado IV: flexión del tronco entre 71-90; *Prueba de Wilcoxon.

Nota: un paciente del grupo experimental abandono el tratamiento por hipotensión.

flexión del tronco, según las mediciones con el goniómetro de grado II y III al inicio del tratamiento y evolucionar al grado IV a las 4 semanas después de haber iniciado el mismo, lo que se demuestra con alta significancia estadística $(\mathrm{p}=0,0000793)$.

En la tabla XIII se muestran los datos relativos al inicio del tratamiento en los pacientes que recibieron la infiltración intramuscular con oxígeno ozono más tratamiento farmacológico y rehabilitador; estos presentaron un porcentaje de incapacidad funcional intensa de 12 pacientes y discapacidad de 5 pacientes, de los cuales, a las 2 semanas después de haber iniciado el tratamiento, el 83,4\% de los primeros y el $60 \%$ de los segundos evolucionaron a la categoría de mínimo porcentaje de incapacidad funcional.

En la tabla XIV se observa que al inicio del tratamiento los pacientes que recibieron tratamiento farmacológico 
TABLA XII. EFICACIA DEL ESQUEMA DE TRATAMIENTO EN EL GRUPO CONTROL AL INICIO Y A LAS 4 SEMANAS POSTERIORES AL TRATAMIENTO. COMPARACIÓN DE LAS CATEGORÍAS DE GRADOS DE FLEXIÓN DEL TRONCO ANTES Y DESPUÉS DE LA INTERVENCIÓN TERAPÉUTICA

Categorías del GFT 4 semanas después de la aplicación del tratamiento

\begin{tabular}{|c|c|c|c|c|c|c|}
\hline $\begin{array}{l}\text { Categorías del GFT al } \\
\text { inicio de la aplicación } \\
\text { del tratamiento }\end{array}$ & & Grado II & Grado III & Grado IV & Total & $p^{*}$ \\
\hline \multirow{2}{*}{ Grado II } & $\mathrm{n}$ & & 1 & 4 & 5 & $0,000532 *$ \\
\hline & $\%$ & & 20,0 & 80,0 & 100,0 & \\
\hline \multirow{2}{*}{ Grado III } & $\mathrm{n}$ & - & 2 & 9 & 11 & \\
\hline & $\%$ & - & 18,2 & 81,8 & 100,0 & \\
\hline \multirow{2}{*}{ Grado IV } & $\mathrm{n}$ & - & - & 5 & 5 & \\
\hline & $\%$ & - & - & 100,0 & 100,0 & \\
\hline \multirow{2}{*}{ Total } & $\mathrm{n}$ & 1 & 5 & 14 & 20 & \\
\hline & $\%$ & 5,0 & 25,0 & 75,0 & 100,0 & \\
\hline
\end{tabular}

Fuente: Formato de Recolección de Datos; Grado I: flexión del tronco entre 0-30; Grado II: flexión del tronco entre 31-50; Grado III: flexión del tronco entre 51-70; Grado IV: flexión del tronco entre 71-90; *Prueba de Wilcoxon.

Nota: un paciente del grupo control abandono el tratamiento por domicilio lejano.

TABLA XIII. EFICACIA DEL ESQUEMA DE TRATAMIENTO EN EL GRUPO EXPERIMENTAL AL INICIO Y A LAS 2 SEMANAS POSTERIORES AL TRATAMIENTO. COMPARACIÓN DE LAS CATEGORÍAS DEL PORCENTAJE DE INCAPACIDAD FUNCIONAL ANTES Y DESPUÉS DE LA INTERVENCIÓN TERAPÉUTICA

Categorías del \% IF 2 semanas después de la aplicación del tratamiento

Categorias del \% IF

previo a la aplicación

del tratamiento

Minima Moderada Discapacidad Total $p^{*}$

\begin{tabular}{|c|c|c|c|c|c|c|}
\hline \multirow{2}{*}{ Moderada } & $\mathrm{n}$ & 5 & - & - & 5 & $0,0000749^{*}$ \\
\hline & $\%$ & 100,0 & - & - & 100,0 & \\
\hline \multirow{2}{*}{ Intensa } & $\mathrm{n}$ & 10 & 1 & 1 & 12 & \\
\hline & $\%$ & 83,4 & 8,3 & 8,3 & 100,0 & \\
\hline \multirow{2}{*}{ Discapacidad } & $\mathrm{n}$ & 3 & 1 & 1 & 5 & \\
\hline & $\%$ & 60,0 & 20,0 & 20,0 & 100,0 & \\
\hline \multirow{2}{*}{ Total } & $\mathrm{n}$ & 18 & 2 & 2 & 22 & \\
\hline & $\%$ & 81,8 & 9,1 & 9,1 & 100,0 & \\
\hline
\end{tabular}

Fuente: Formato de Recolección de Datos; IF: incapacidad funcional; Mínima (0-19 puntos); Moderada (20-39 puntos); Intensa (40-59 puntos); Discapacidad (60-79 puntos); *Prueba de Wilcoxon.

y rehabilitador, presentaron un porcentaje de incapacidad funcional moderada de 10 pacientes y de discapacidad 4 pacientes, de los cuales, a las 2 semanas después de haber iniciado el tratamiento el $100 \%$ de los primeros y ninguno $(0 \%)$ de los segundos evolucionaron a la categoría de mínimo porcentaje de incapacidad funcional
En la tabla XV se aprecia que al inicio del tratamiento en los pacientes que recibieron la infiltración intramuscular con oxígeno ozono más tratamiento farmacológico y rehabilitador, presentaron un porcentaje de incapacidad funcional intensa 12 pacientes y de discapacidad 5 pacientes, de los cuales a las 4 semanas después de haber iniciado 
TABLA XIV. EFICACIA DEL ESQUEMA DE TRATAMIENTO EN EL GRUPO CONTROL AL INICIO Y A LAS 2 SEMANAS POSTERIORES AL TRATAMIENTO. COMPARACIÓN DE LAS CATEGORÍAS DEL PORCENTAJE DE INCAPACIDAD FUNCIONAL ANTES Y DESPUÉS DE LA INTERVENCIÓN TERAPÉUTICA

Categorías del \% IF 2 semanas después de la aplicación del tratamiento

\begin{tabular}{|c|c|c|c|c|c|c|c|}
\hline $\begin{array}{l}\text { Categorías del } \\
\% \text { IF previo a la } \\
\text { aplicación del } \\
\text { tratamiento }\end{array}$ & & Mínima & Moderada & Intensa & Discapacidad & Total & $p^{*}$ \\
\hline \multirow{2}{*}{ Mínima } & $\mathrm{n}$ & 2 & - & - & - & 2 & $0,0000221 *$ \\
\hline & $\%$ & 100,0 & - & - & - & 100,0 & \\
\hline \multirow{2}{*}{ Moderada } & $\mathrm{n}$ & 10 & - & - & - & 10 & \\
\hline & $\%$ & 100,0 & - & - & - & 100,0 & \\
\hline \multirow{2}{*}{ Intensa } & $\mathrm{n}$ & - & 5 & - & - & 5 & \\
\hline & $\%$ & - & 100,0 & - & - & 100,0 & \\
\hline \multirow{2}{*}{ Discapacidad } & $\mathrm{n}$ & - & - & 3 & 1 & 4 & \\
\hline & $\%$ & - & - & 75,0 & 25,0 & 100,0 & \\
\hline \multirow{2}{*}{ Total } & $\mathrm{n}$ & 12 & 5 & 3 & 1 & 21 & \\
\hline & $\%$ & 57,1 & 23,8 & 14,3 & 4,8 & 100,0 & \\
\hline
\end{tabular}

Fuente: Formato de Recolección de Datos; IF: Incapacidad funcional; Minima (0-19 puntos); Moderada (20-39 puntos); Intensa (40-59 puntos); Discapacidad (60-79 puntos); *Prueba de Wilcoxon.

TABLA XV. EFICACIA DEL ESQUEMA DE TRATAMIENTO EN EL GRUPO EXPERIMENTAL AL INICIO Y A LAS 4 SEMANAS POSTERIORES AL TRATAMIENTO. COMPARACIÓN DE LAS CATEGORÍAS DEL PORCENTAJE DE INCAPACIDAD FUNCIONAL ANTES Y DESPUÉS DE LA INTERVENCIÓN TERAPÉUTICA

Categorías del \% IF 4 semanas después de la aplicación del tratamiento

\begin{tabular}{|c|c|c|c|c|c|}
\hline $\begin{array}{l}\text { Categorias del \% IF } \\
\text { previo a la aplicación del } \\
\text { tratamiento }\end{array}$ & & Mínima & Discapacidad & Total & $p^{*}$ \\
\hline \multirow{2}{*}{ Moderada } & $\mathrm{n}$ & 4 & - & 4 & \multirow[t]{8}{*}{$0,0000987^{*}$} \\
\hline & $\%$ & 100,0 & - & 100,0 & \\
\hline \multirow{2}{*}{ Intensa } & $\mathrm{n}$ & 11 & 1 & 12 & \\
\hline & $\%$ & 91,7 & 8,3 & 100,0 & \\
\hline \multirow{2}{*}{ Discapacidad } & $\mathrm{n}$ & 4 & 1 & 5 & \\
\hline & $\%$ & 80,0 & 20,0 & 100,0 & \\
\hline \multirow{2}{*}{ Total } & $\mathrm{n}$ & 19,0 & 2,0 & 21 & \\
\hline & $\%$ & 90,5 & 9,5 & 100,0 & \\
\hline
\end{tabular}

Fuente: Formato de Recolección de Datos; IF: Incapacidad funcional; Minima (0-19 puntos); Moderada (20-39 puntos); Intensa (40-59 puntos); Discapacidad (60-79 puntos); *Prueba de Wilcoxon.

Nota: un paciente del grupo experimental abandono el tratamiento por hipotensión. 
el tratamiento, el $91,7 \%$ de los primeros y el $80 \%$ de los segundos evolucionaron a la categoría de mínimo porcentaje de incapacidad funcional.

En la tabla XVI se evidencia que al inicio del tratamiento los pacientes que recibieron tratamiento farmacológico y rehabilitador presentaron un porcentaje de incapacidad funcional moderada de 10 pacientes y de discapacidad 3 pacientes por abandono de uno durante la investigación. De estos, a las 4 semanas después de haber iniciado el tratamiento, el $100 \%$ de los primeros y ninguno (0\%) de los segundos evolucionaron a la categoría de mínimo porcentaje de incapacidad funcional.

Examinando los resultados de las tablas XV y XVI vemos que el tratamiento del grupo experimental fue más eficaz en un $90,5 \%$ de los pacientes (el tratamiento del grupo control lo fue en un $70 \%$ de los pacientes), así como en disminuir el porcentaje de incapacidad funcional de discapacidad intensa y moderada según la escala de Oswestry al inicio del tratamiento y evolucionar al porcentaje de discapacidad mínima las 4 semanas después de haber iniciado el tratamiento, lo que se demuestra con alta significancia estadística ( $\mathrm{p}=0,0000987)$.

\section{Efectos colaterales presentados en el grupo experimental, tras la aplicación de ozono $\left(\mathrm{O}_{2} \mathrm{O}_{3}\right)$}

En cuanto a los efectos colaterales, todos los pacientes del grupo experimental en el momento de la infiltración con oxígeno ozono intramuscular a nivel paravertebral lumbar y en puntos miofasciales reportaron la presencia de ardor localizado, con una duración de 15 minutos, más intenso durante las primeras infiltraciones. A medida que fueron transcurriendo las semanas y aumentando el número de infiltraciones, desapareció dicha molestia. Sólo se reportaron 2 casos de hipotensión, razón por la cual uno de estos pacientes no continuó con el tratamiento.

\section{DISCUSIÓN}

El estudio mostró que el dolor lumbar crónico se presentó con preferencia en el sexo femenino en un 60,5\%, en edades comprendidas entre los 30 y 60 años en un $95,3 \%$, y en menor proporción en individuos de los grupos de edad mayores de 60 y menores de 21 años. Similares son los resultados encontrados por Humbría (13) de un estudio realizado en 334 pacientes con dolor lumbar, en donde predominó el sexo femenino en un $67 \%$ y la población entre 30 y 60 años de edad en $76 \%$.

Kelsey y White (14) señalaron que la patología lumbar aguda es la causa más común de limitación de la actividad en pacientes menores de 45 años, en cambio los resultados de este estudio confirman que la mayoría de los pacientes manifiestan edades iguales o superiores a los 45 años, sugiriendo que en caso de dolor lumbar crónico y lumbociatalgia crónica, la edad de presentación clínica es más tardía.

TABLA XVI. EFICACIA DEL ESQUEMA DE TRATAMIENTO EN EL GRUPO CONTROL AL INICIO Y A LAS 4 SEMANAS POSTERIORES AL TRATAMIENTO. COMPARACIÓN DE LAS CATEGORÍAS DEL PORCENTAJE DE INCAPACIDAD FUNCIONAL ANTES Y DESPUÉS DE LA INTERVENCIÓN TERAPÉUTICA

Categorías del \% IF 4 semanas después de la aplicación del tratamiento

\begin{tabular}{|c|c|c|c|c|c|c|}
\hline $\begin{array}{l}\text { Categorias del \% IF } \\
\text { previo a la aplicación } \\
\text { del tratamiento }\end{array}$ & & Minima & Moderada & Discapacidad & Total & $p^{*}$ \\
\hline \multirow{2}{*}{ Mínima } & $\mathrm{n}$ & 2 & - & - & 2 & $0,000130^{*}$ \\
\hline & $\%$ & 100,0 & - & - & 100,0 & \\
\hline \multirow{2}{*}{ Moderada } & $\mathrm{n}$ & 10 & - & - & 10 & \\
\hline & $\%$ & 100,0 & - & - & 100,0 & \\
\hline \multirow{2}{*}{ Intensa } & $\mathrm{n}$ & 2 & 3 & - & 5 & \\
\hline & $\%$ & 40,0 & 60,0 & - & 100,0 & \\
\hline \multirow{2}{*}{ Discapacidad } & $\mathrm{n}$ & - & 2 & 1 & 3 & \\
\hline & $\%$ & - & 66,7 & 33,3 & 100,0 & \\
\hline \multirow{2}{*}{ Total } & $\mathrm{n}$ & 14 & 5 & 1 & 20 & \\
\hline & $\%$ & 70,0 & 25,0 & 5,0 & 100,0 & \\
\hline
\end{tabular}


Existen ocupaciones laborales con tendencia a presentar mayor dolor lumbar (15); esta afirmación coincide con los resultados de la actual investigación donde la enfermería es una de las profesiones que más consultó, ocupando el primer lugar en un 18,5\%.

Gunnar, Andersson y Gregory (16) refieren que el 97\% de las lumbalgias inespecíficas se atribuyen a distensiones o esguinces de tejidos blandos, similar a los hallazgos de este trabajo, donde se evidencia el predominio y la importancia de la patología muscular como etiología predominante, presentándose en el $100 \%$ de los pacientes del estudio, ya sea como causa única de dolor lumbar crónico en un $39,5 \%$ o acompañando a otras patologías asociadas en el restante $60,5 \%$ de los pacientes. A diferencia de Insausti (4) y Rodríguez (7), quienes describen que los músculos asociados con mayor frecuencia al dolor lumbar son el cuadrado lumbar, psoas lumbar y piriforme, en el estudio se mostró que en primer lugar estaban involucrados los paravertebrales, seguidos del cuadrado lumbar, piriforme y la asociación de músculos en miembros inferiores en casos de lumbociatalgia crónica. Los puntos gatillos localizados en el área paravertebral, correspondieron a los niveles L4-L5 en $55 \%$, L5-S1 en $30 \%$ y otras áreas en $15 \%$, coincidiendo con Paoloni y cols. (10) que los ubicaron en los niveles L4-L5 en $65 \%$ y L5-S1 en $25 \%$.

Entre otras causas de dolor lumbar crónico se encontró el síndrome facetario con un 25,6\%, ubicándose dentro del rango como lo describen Dreyer y Dreyfuss (17) del 15 al $40 \%$ para esta patología. Stevenson y Anderson (18) demostraron que un $40 \%$ de los pacientes que presentan lumbociatalgia se asocia con la presencia de hernia discal lumbar, mostrándose en relación con el $35 \%$ de los pacientes de la investigación.

Otros autores refieren que los pacientes con dolor lumbar a causa de síndrome doloroso miofascial evolucionan peor en la disminución de la intensidad del dolor en comparación con los pacientes que presentan grados de discopatías (8), siendo estos resultados contrarios a los reportados en este estudio, donde se demostró que la técnica de infiltración intramuscular con ozono a nivel paravertebral y en puntos gatillos revierte la sintomatología en la mayoría de los pacientes. Es de hacer notar que 2 de los pacientes del grupo experimental y 1 paciente del grupo control (en total 3 pacientes que representan el $7 \%$ ) no mejoraron al final del tratamiento al no presentar disminución en la intensidad del dolor, en la incapacidad funcional ni aumentar la flexión del tronco, debido a lumbociatalgia crónica por extrusión discal central transligamentosa, condicionando una estenosis central del canal vertebral que necesitó posterior resolución quirúrgica.

Los resultados satisfactorios en los pacientes con estenosis espinal lateral de tipo receso lateral se lograron porque las estenosis eran de tejido blando interpuesto, de tipo protrusión discal asimétrica.
Se puede resaltar entre los hallazgos de la investigación, que la intervención terapéutica utilizando la técnica de infiltración con ozono intramuscular paravertebral lumbar $\mathrm{y}$ en puntos gatillos junto al tratamiento farmacológico y rehabilitador en el manejo de los pacientes con dolor lumbar crónico y lumbociatalgia crónica, resultó más eficaz que la modalidad de tratamiento combinado farmacológico y rehabilitador, demostrado con una significancia estadística $(\mathrm{p}<0,05)$ al disminuir la intensidad del dolor en un $90,5 \%$, la incapacidad funcional en el 90,5\% y al aumentar los grados de flexión del tronco en $85,7 \%$ comparado en un $40,0,70$ y $75 \%$, respectivamente, en pacientes que cursaron con síndrome doloroso miofascial, síndrome facetario, protrusiones discales y estenosis de receso lateral, a las 4 semanas posteriores a los inicios de tratamientos.

Paoloni y cols. (10), en 2009, administraron infiltraciones con ozono intramuscular en el área paravertebral lumbar durante 5 semanas y, aunque mostraron resultados satisfactorios, estos carecen de repercusión, ya que los pacientes incluidos en el mismo presentaban sólo dolor lumbar y lumbociatalgia de tipo agudo cuya sintomatología sabemos es autolimitada. En contraste, los resultados beneficiosos de la actual investigación se consiguen en pacientes con dolor lumbar crónico y lumbociatalgia crónica.

Moretti y cols. (19) trabajaron en pacientes con dolor lumbar crónico, con monoterapia a expensas de la infiltración intramuscular paravertebral con ozono, observando resultados poco favorables ya que el $50 \%$ de los pacientes necesitaron la administración de un segundo ciclo de 3 semanas adicionales para disminuir la intensidad del dolor. En cambio, con el uso del ozono paravertebral lumbar y en puntos gatillos, junto al tratamiento multimodal, se demostró que sólo el 9,5 \% de los pacientes no disminuyeron la intensidad del dolor según la EVA en el primer y único ciclo de 4 semanas de tratamiento.

Arena y Savoca (20) manejaron pacientes con dolor lumbar y lumbociatalgia crónica por hernia discal lumbar con tratamiento combinado por medio de ozono paravertebral de 15-20 infiltraciones, ejercicios, escuela de espalda y medios físicos, encontrando la remisión completa del dolor y la mejor movilidad del tronco con un porcentaje de $75,9 \%$ a las 8-10 semanas; contrario a esa investigación, en este estudio se incorporó el tratamiento farmacológico y se infiltraron otros puntos gatillos adicionales a los paravertebrales, obteniendo la remisión completa del dolor y la mejor movilidad del tronco en el 90,5\% de los pacientes con 8 infiltraciones al cumplir las 4 semanas del tratamiento. Estos mismos autores afirman que el uso de terapias integradas puede lograr un aumento significativo en las tasas de remisión de síntomas.

A diferencia de lo que exponen Ansede, Contreras y Pérez (21), quienes refieren la aparición de cefaleas con la aplicación del ozono paravertebral lumbar, en la investigación el $100 \%$ de los pacientes refieren ardor transitorio 
en el área aplicada e hipotensión en el 8,6\% $(\mathrm{n}=2)$ de los pacientes, la cual puede llegar a disminuir si en las infiltraciones iniciales de ozono se utilizan concentraciones menores.

\section{CONCLUSIONES}

- Se evidencia que en pacientes con diagnóstico de lumbociatalgia crónica por discopatía de tipo extrusión transligamentosa, que condiciona una estenosis central del canal vertebral, no se obtiene una respuesta satisfactoria con infiltración paravertebral lumbar y en puntos gatillos, a diferencia de los hallazgos beneficiosos que se alcanzan en los pacientes con dolor lumbar y lumbociatalgia crónica con diagnósticos de síndrome doloroso miofascial aislado (ubicado frecuentemente en la musculatura paravertebral, cuadrado lumbar, piriforme, gemelos, isquiotibiales y aductores de cadera) o asociado al síndrome facetario, protrusión discal y síndrome de receso lateral, por lo que en estos casos, realizar el estudio de imagen de resonancia magnética lumbosacra, se convierte en un aliado diagnóstico para priorizar el tratamiento según el caso de cada paciente.

- El tratamiento del grupo experimental que incluyó la infiltración con ozono junto al tratamiento farmacológico y rehabilitador resulta más eficaz que la modalidad combinada de tratamiento farmacológico y rehabilitador, demostrado con una alta significancia estadística $(\mathrm{p}<0,05)$ al disminuir la intensidad del dolor a un EVA de bueno en el 90,5\%, disminuir la incapacidad funcional a mínima en el $90,5 \%$ y en aumentar los grados de flexión del tronco a la máxima flexión en un 85,7\%, comparado con los resultados del grupo control de 40,70 y $75 \%$ respectivamente a las 4 semanas, posteriores al inicio de los tratamientos; esta situación disminuye la ingesta de medicamentos analgésicos, el ausentismo laboral y mejora el cumplimiento en las actividades de la vida diaria.

- Es importante que se conozca la patología del síndrome doloroso miofascial ya que posiblemente un alto porcentaje de lumbalgias inespecíficas se atribuyan a esta causa.

- Se recomienda utilizar la técnica de infiltración con ozono a nivel paravertebral lumbar y en puntos gatillos ya que, además de desactivar estos últimos con el solo paso de la aguja al igual que la técnica de punción seca, el ozono adicionalmente provoca vasodilatación, un efecto analgésico y antiinflamatorio con relajación de los tejidos comprometidos (fascia $\mathrm{y}$ vientre muscular) en un corto tiempo.

- La técnica de infiltración con ozono a nivel paravertebral lumbar y en puntos gatillos en pacientes con dolor lumbar crónico y lumbociatalgia crónica no se recomienda como monoterapia, sino como una herramienta más a utilizar dentro del arsenal terapéutico conservador en esta patología.

- Concluimos en que el dolor lumbar es tan antiguo como la aparición del hombre, lo que ha cambiado es la forma de entenderlo y manejarlo (22).

CORRESPONDENCIA:

Erlinda Naydú Silva Jiménez

e-mail: erlinda803@hotmail.com

\section{BIBLIOGRAFÍA}

1. Sánchez I. Manual SERMEF de rehabilitación y medicina física. En: Flórez M, García F. Dolor Lumbar. $1^{\mathrm{a}}$ ed. Madrid-España: Panamericana; 2006. p. 387-99.

2. Salinas F, Lugo L, Restrepo R. Rehabilitación en salud. Una mirada necesaria. En: Arias J. Dolor Lumbar. $2^{\mathrm{a}}$ ed. Medellín-Colombia: Editorial Universidad de Antioquia; 2008. p. 221-41.

3. AVED Asociación venezolana para el estudio del dolor. Concenso venezolano sobre dolor neuropático. En: Franco L, Jreige A, Krivoy J, Fernández A, Mártinez M, Romero L. Dolor Lumbar. Caracas - Venezuela: Editorial normacolor; 2007. p. 95-125.

4. Insausti J. Lumbalgia inespecífica: en busca del origen del dolor. Reumatol Clin 2009;5(S2):19-26.

5. Peña JL, Peña, C, Brieva, P, Pérez M, Humbria Mendiola. Fisiopatología de la lumbalgia. Rev EspReumatol 2002;29:483-8.

6. Simons D, Travell J, Simons L. Myofascial pain and dysfunction. 2nd ed. Baltimore: Williams \&Wikins; 1999.

7. Rodríguez M, Aldaya C, Martínez G, Gil E. Síndrome de dolor miofascial de la cintura pélvica. Rev Soc Esp Dolor 2007; 14:358-68.

8. Cassisi J E, Sypert GW, Lagana L, Friedman EM, Robinson ME. Pain, disability, and psychological functioning in chronic low back pain subgroups: Myofascial versus herniated disc syndrome. Neurosurgery 1993;33:379-85.

9. Menéndez S, González R, Ledea O, Hernández F, León O, Díaz M. Ozono aspectos básicos y aplicaciones clínicas. $1^{\mathrm{a}}$ ed. Cuba: Editorial CENICM; 2008.

10. Paoloni M, Di Sante L, Cacchio A, Apuzzo D, Marotta S, Razzano M, et al. Intramuscular oxygen-ozone therapy in the treatment of acute back pain with lumbar disc herniation. Spine 2009;13:1337-44.

11. Borrelli E. Mechanism of action of oxygen ozone therapy in the treatment of disc herniation and low back pain. Acta Neurochir Suppl 2011;108:123-5.

12. Coclite D, Napoletano A, Barbina D. Conferenza di consenso ossigeno-ozono terapia nel trattamento delle lombosciatalgie da ernia discale con tecnica iniettiva intramuscolare paravertebrale. Istituto Superiore di Sanità. Roma, 20 novembre 2006. Roma: Istituto Superiore di Sanita; 2008. (Rapporti ISTISAN 08/9).

13. Humbría A. Consulta monográfica de columna lumbar. Protocolo de investigación clínica, ¿cómo son los pacientes con lumbalgia crónica inespecífica? Rev Esp Reumatol 2004;31(8):453-61. 
14. Kelsey J, White A. Epidemiology and impact of low-back pain. Spine 1980;5:133-7.

15. Bordini L, De Vito G, Molteni G, Boccardi S. The epidemiology of musculoskeletal changes due to biomechanical overload of the spine in the manual lifting of patients. Med Lav 1999;90(2):103-16.

16. Gunnar B, Andersson M, Gregory G. Orthopaedic Knowledge Update columna vertebral. In: Beaty JH, editor. Trastornos degenerativos lumbares. Barcelona; 2000. p. 63-79.

17. Dreyer F, Dreyfuss P. Low back pain and the zygapophysial (facet) joints. Arch Phys Med Rehabil 1996;77(3):290300.

18. Stevenson H, Anderson G. Low-back pain in 40-to 47-year old men: Work history and work environment factors. Spine 1983;8:272-6.
19. Moretti B, Lanzisera R, Pesce V, Moretti L, Patella, S, Simone V. Iltrattamento con O2-O3 paravertebral en elle lombalgie meccaniche. Rivista Italiana di Ossigeno-Ozonoterapia 2003;2:139-46.

20. Arena M, Savoca G. Infiltrazione percutánea paravertebrale di O2-O3. Trattament integrati nella terapia delle ernie e dei conflitti discoarticolari a sede lombare. Riv Italiana di Ossigeno-Ozonoterapia 2003;2:39-44.

21. Ansede J, Contreras J, Pérez S. Estudio prospectivo y aleatorizado en pacientes con lumbalgias o lumbociatalgias tratados con ozonoterapia. Patología del Aparato Locomotor 2007;5(1):46-54.

22. Allan D, Waddell G. An historical perspective on low back pain and disability. Acta Orthopaedica Scandinavica 1989;60:1-23. 